\title{
Screening for primary creatine deficiencies in French patients with unexplained neurological symptoms
}

David Cheillan ${ }^{1 \dagger}$, Marie Joncquel-Chevalier Curt ${ }^{2 \dagger}$, Gilbert Briand ${ }^{2,3}$, Gajja S Salomons ${ }^{4}$, Karine Mention-Mulliez ${ }^{5}$, Dries Dobbelaere ${ }^{5}$, Jean-Marie Cuisset ${ }^{6}$, Laurence Lion-François ${ }^{7}$, Vincent Des Portes ${ }^{7}$, Allel Chabli ${ }^{8}$, Vassili Valayannopoulos ${ }^{9}$, Jean-François Benoist ${ }^{10}$, Jean-Marc Pinard ${ }^{11}$, Gilles Simard ${ }^{12}$, Olivier Douay ${ }^{12}$, Kumaran Deiva ${ }^{13}$, Alexandra Afenjar ${ }^{14}$, Delphine Héron ${ }^{15}$, François Rivier $^{16}$, Brigitte Chabrol ${ }^{17}$, Fabienne Prieur ${ }^{18}$, François Cartault ${ }^{19}$, Gaëlle Pitelet ${ }^{20}$, Alice Goldenberg ${ }^{21}$, Soumeya Bekri² ${ }^{22}$, Marion Gerard ${ }^{23}$, Richard Delorme ${ }^{24}$, Marc Tardieu ${ }^{25}$, Nicole Porchet ${ }^{2}$, Christine Vianey-Saban ${ }^{1}$ and Joseph Vamecq ${ }^{2,26^{*}}$

\begin{abstract}
A population of patients with unexplained neurological symptoms from six major French university hospitals was screened over a 28-month period for primary creatine disorder (PCD). Urine guanidinoacetate (GAA) and creatine: creatinine ratios were measured in a cohort of 6,353 subjects to identify PCD patients and compile their clinical, ${ }^{1} \mathrm{H}-\mathrm{MRS}$, biochemical and molecular data. Six GAMT [N-guanidinoacetatemethyltransferase (EC 2.1.1.2)] and 10 X-linked creatine transporter (SLC6A8) but no AGAT (GATM) [L-arginine/glycine amidinotransferase (EC 2.1.4.1)] deficient patients were identified in this manner. Three additional affected sibs were further identified after familial inquiry (1 brother with GAMT deficiency and 2 brothers with SLC6A8 deficiency in two different families). The prevalence of PCD in this population was $0.25 \%$ (0.09\% and 0.16\% for GAMT and SLC6A8 deficiencies, respectively). Seven new PCD-causing mutations were discovered (2 nonsense [c.577C > T and c.289C > T] and 1 splicing $[c .391+15 G>T$ ] mutations for the GAMT gene and, 2 missense [c.1208C > A and c.926C > A], 1 frameshift [c.930delG] and 1 splicing [c.1393-1G > A] mutations for the SLC6A8 gene). No hot spot mutations were observed in these genes, as all the mutations were distributed throughout the entire gene sequences and were essentially patient/family specific. Approximately one fifth of the mutations of SLC6A8, but not GAMT, were attributed to neo-mutation, germinal or somatic mosaicism events. The only SLC6A8-deficient female patient in our series presented with the severe phenotype usually characterizing affected male patients, an observation in agreement with recent evidence that is in support of the fact that this $X$-linked disorder might be more frequent than expected in the female population with intellectual disability.
\end{abstract}

\footnotetext{
* Correspondence: joseph.vamecq@inserm.fr

${ }^{\dagger}$ Equal contributors

${ }^{2}$ Département de Biochimie et Biologie Moléculaire, Laboratoire

d'Hormonologie, Métabolisme-Nutrition \& Oncologie (HMNO)-Centre de

Biologie et Pathologie (CBP) Pierre-Marie Degand, CHRU Lille, Lille 59037,

France

${ }^{26}$ Inserm, Laboratoire Externe, Département du Prof. Nicole Porchet, HMNO, Centre de Biologie et Pathologie (CBP) Pierre-Marie Degand, CHRU Lille, Lille 59037, France

Full list of author information is available at the end of the article
} 


\section{Introduction}

Creatine is a physiological compound that was first isolated by the French chemist Eugène Chevreul in the 1830s [1]. It is supplied to the body both via endogenous biosynthesis and the diet [2-4]. Its name (the Greek 'kreas' means meat) originates from its abundance in muscle in which its energetic role has previously been documented [5-8]. Creatine can be phosphorylated by creatine kinases, leading to the storage of a high-energy phosphate bond of ATP in the form of phosphocreatine [3]. Upon demand, and due to the reversible activity of creatine kinases which can transform ADP back into ATP, phosphocreatine can release the stored energy [3]. This creatine/phosphocreatine cycle represents a vital reserve of energy in tissues with high energetic needs such as muscles and the brain [3]. In recent years, the physiological role of brain creatine has been extended to include the modulatory control of neurotransmission which, in addition to guanidinoacetate (GAA) toxicity, has improved our understanding of brain function and its severe impairment in disorders affecting creatine biosynthesis and transport [9-12].

The first step of creatine biosynthesis occurs essentially but not exclusively in the kidney and involves the transfer of an amidino group from arginine to glycine by the enzyme L-arginine/glycine amidinotransferase (AGAT) (EC 2.1.4.1) to form GAA and L-ornithine. Subsequently, $\mathrm{N}$-guanidinoacetatemethyltransferase (GAMT) (EC 2.1.1.2) catalyzes the transfer of a methyl group from $S$-adenosyl methionine (SAM) on GAA. This second step occurs notably in the liver and leads to the formation of creatine and $S$-adenosylhomocysteine (SAH) [3]. Once synthesized, creatine may be released into the bloodstream. It can be internalized by cells via a specific plasma membrane $\mathrm{Na}^{+} / \mathrm{Cl}^{-}$dependent creatine transporter (SLC6A8) and stored as phosphocreatine [3]. Finally, about $1.7 \%$ of the creatine/phosphocreatine pool undergoes spontaneous degradation each day forming creatinine that is excreted in urine [3]. Urinary excretion of creatinine is a function of body muscle mass and in practice is a useful marker of kidney function. Little or no creatine is taken up by the brain from the systemic bloodstream because the blood brain barrier is practically impermeable to creatine. The brain must therefore secure most of its requirements in creatine by endogenous synthesis $[10,11,13]$. The crucial role of SLC6A8, which has been reviewed recently [14], provides ground for understanding how its deficiency results in a creatine collapse in brain.

Extracerebral and intracerebral routes for creatine supplies concur and their respective contributions differ in the immature (i.e. fetal and perinatal) and mature brain. In the immature brain, the extracerebral supply is favored due to a better efficacy of SLC6A8 in the bloodbrain barrier (BBB). This means that extraction of creatine from the blood is predominant over intracerebral creatine biosynthesis which is physiologically limited by low GAMT activity in the immature brain [14]. SLC6A8 deficiency affects the immature brain creatine content through impairment of BBB uptake. In the mature brain, the contribution of BBB SLC6A8 becomes physiologically lower and intracerebral biosynthesis becomes the major pathway for the supply of brain creatine [14]. SLC6A8 deficiency therefore affects the mature brain creatine content through impairment of intracerebral creatine biosynthesis as a result of reduced SLC6A8-driven transfer of GAA from brain GAA to creatine-synthesizing cells. Differences existing between the extracellular/intracellular routes for the brain creatine supply explain why creatine supplementation is more efficient in two other creatine disorders, AGAT and GAMT deficiencies, when given at a presymptomatic stage of the disease when BBB uptake of creatine is physiologically optimal $[14,15]$. An illustrated account of creatine biosynthesis and transport is provided in the Additional file 1.

Primary creatine deficiency disorders (PCD) are a new class of inborn errors of metabolism to which belong AGAT (OMIM 602360) and GAMT (OMIM 601240) deficiencies, both inherited as an autosomal recessive trait, and SLC6A8 [CT1, CRTR] deficiency (OMIM 300036), an X-linked disorder [10-12,16-20]. The three disorders share a relative reduction of brain creatine and phosphocreatine levels [19], and accordingly, the clinical presentation is predominated by neurological signs [10-12,16-20]. However, these disorders still remain under-diagnosed whether considered together [16-20] or individually (AGAT [21-25], GAMT [26,27] or CRTR [28-33] deficiency).

In this paper we present the results of a large retrospective study of urinary screening for PCD on 6,353 patients in whom the etiology of the underlying neurological disease was unknown at the time of urinary analysis of creatine and its metabolites.

\section{Patients and methods}

We retrospectively collected the metabolic results of urinary screening for PCD (GAA and creatine:creatinine ratios) from 6,353 patients with neurological disease of unknown origin $(4,426$ male patients and 1,927 female patients) hospitalized in six French university hospitals (Angers, Lille, Lyon and Paris (Hôpital Necker Enfants Malades, Hôpital Robert Debré and Hôpital Raymond Poincaré)) over a period of 28 months. Data with regard to GAA and creatine levels in plasma were also collected when available. For all patients with an abnormal result, a second sample was analyzed after a 24-hour diet devoid of meat and fish according to the recommendation of Arias et al. [34]. A patient was considered 
"at risk of PCD" (AGAT, GAMT or SLC6A8 deficiency) when the biochemical abnormality was confirmed on this second sample. Subsequently a molecular study and/or a functional test were performed to confirm the diagnosis. For the genetic study, informed consent was obtained from each patient or from their parents if probands were under 18 . For patients with a confirmed diagnosis of PCD, extensive clinical data were collected, including familial, obstetrical and personal histories, main symptoms having led to consult, the specialty of the physician who requested metabolic investigations, the patient's age at diagnosis, clinical signs present at diagnosis and results of ${ }^{1} \mathrm{H}$-MRS exploration. All patients with other diagnosed conditions (in particular, urea cycle disorders and remethylation defects) were discarded from the study.

\section{Methods for metabolite measurements}

Creatine and GAA levels were measured in urine and plasma by tandem mass spectrometry (LC-MS/MS) using stable isotopes as internal standards $\left({ }^{13} \mathrm{C}_{2}\right.$-GAA and ${ }^{2} \mathrm{H}_{3}$-creatine) according to a method described previously [35]. Urinary creatinine was measured using LC-MS/MS using a specific internal standard $\left({ }^{2} \mathrm{H}_{3^{-}}\right.$ creatinine), except in two university hospitals in Paris (Necker Enfants Malades and Robert Debré) where this measurement was performed using the colorimetric Jaffé method [36,37]. The control values and age groups used for plasma and urine GAA and creatine concentrations were those published by Verhoeven et al. [38].

\section{Measurement of protein activities}

GAMT activity was determined in lymphoblasts by assaying the transfer of methyl groups from $\left[{ }^{2} \mathrm{H}_{3}\right]-\mathrm{S}$ adenosylmethionine to ${ }^{13} \mathrm{C}_{2}$-labelled GAA through the production of $\left[{ }^{13} \mathrm{C}_{2}{ }^{2} \mathrm{H}_{3}\right]$-creatine quantitated by an isotope dilution electrospray tandem spectrometry assay [39].

Creatine transporter activity was assayed by determining the intracellular incorporation of creatine in cultured skin fibroblasts as previously described [32].

\section{Genetic studies}

Genomic DNA was isolated from white blood cells or cultured fibroblasts collected from patients after informed consent. The coding regions and adjacent intronic splice sites of the GAMT and SLC6A8 genes were analyzed by direct sequencing. The pathogenicity of previously non-described mutations was predicted using the Alamut v2.02 software (Interactive Biosoftware, Rouen France) and compared to the LOVD database [40].

\section{Results}

Results of the screening for PCD in the French patients with unexplained neurological symptoms

Among the 6,353 patients screened, 16 patients were diagnosed to have PCD: 6 with GAMT deficiency (4 male and 2 female patients) and 10 with SLC6A8 deficiency ( 9 male and 1 female patients). Three additional affected sibs were identified after familial inquiries (1 brother affected with GAMT deficiency and 2 brothers with SLC6A8 deficiency in two different families). Diagnosis was confirmed by genetic analysis in 17 patients. For the other 2 patients (1 with GAMT deficiency and 1 with SLC6A8 deficiency), DNA analysis was rejected by the parents and the diagnosis was confirmed by the absence of a creatine peak in brain ${ }^{1} \mathrm{H}$-MRS, the urinary profile and the deficiency of protein activities. No patients with AGAT deficiency were identified in our cohort. The medical investigations leading to the diagnosis of these pathologies were requested by pediatric neurologists (42\%), geneticists (32\%), pediatricians (10.5\%), adult neurologists (10.5\%) and child psychiatrists (5\%). Clinical descriptions and the results of the diagnostic explorations performed for the index patients and for the affected sibs are summarized in Table 1. The global prevalence of PCD in the screened population was $0.25 \%(16 / 6,353$; CI95: $0.13 \%-0.38 \%)$, the prevalence of GAMT deficiency was $0.09 \%$ (6/6,353; CI95: $0.02 \%$ $0.17 \%$ ) and the prevalence of SLC6A8 deficiency was 0.16\% (12/6,353; CI95: 0.06\% - 0.26\%). Regarding our data, the prevalence of AGAT deficiency would be lower than $1 / 6,353$, i.e. $<0.02 \%$ since no AGAT patient was diagnosed in the present study. The prevalence of PCD was $0.29 \%$ for the male patients (4 GAMT and 9 SLC6A8 deficiencies, 13/4,426; CI95: 0.14\% - 0.46\%) and $0.16 \%$ for the female patients (2 GAMT and 1 SLC6A8 deficiencies, 3/1,927; CI95: 0.0\% - 0.34\%). For the Xlinked disorder SLC6A8 deficiency, the prevalence was $0.20 \%$ for the male patients $(9 / 4,426$; C195: $0.08 \%$ $0.34 \%)$ and $0.05 \%(1 / 1,927$; CI95: $0.0 \%-0.16 \%)$ for the female patients.

\section{Patients with GAMT deficiency}

Seven patients from 6 unrelated families were diagnosed with GAMT deficiency. The sex ratio $(\mathrm{M} / \mathrm{F})$ was 2.5 . Five of the 7 patients were born of a consanguineous union. Most of the patients were of Caucasian origin. Obstetrical history was available for 5 of the 7 patients; pregnancies were normal without pre-term birth. The mean \pm SD values for birth-related parameters were: gestational term, $39.1 \pm 0.5$ weeks; birth weight, $3192 \pm$ $135 \mathrm{~g}$; birth length, $49.4 \pm 1.4 \mathrm{~cm}$ and head circumference, $34.0 \pm 1.2 \mathrm{~cm}$. The predominant clinical signs at diagnosis were intellectual disability $(100 \%)$ and speech delay $(100 \%)$ (for general information on speech 
Table 1 Main clinical, biological, genetic and ${ }^{1} \mathrm{H}-\mathrm{MRS}$ features of the patients affected with PCD

GAMT $(n=7) \quad$ CRTR $(n=12)$

\section{CLINICAL DATA}

Sex

\section{Male}

Female

Ratio (M/F)

Consanguinity

Age of onset (years)

$\leq 2$ years

$>2$ years

$1 / 7$

Age at diagnosis(years)

$\leq 2$ years

[2-5 years]

[5-10 years]

$>10$ years

Main clinical signs

Intellectual disability

Speech delay

Failure to thrive

Hypotonia

Myopathy

Motor delay

Epilepsy

Extrapyramidal signs

Attention deficit

Sleeping disturbances

Agressive behaviour

Autistic behaviour

Signs leading to consult

Combined motor

and speech delay

Epilepsy

Intellectual disability

Autistic behavior

Familial exploration

\section{METABOLIC DATA}

Plasma GAA $(\mu \mathrm{mol} / \mathrm{l})$

$$
<15 \text { years: }[0.35-1.8]^{a}
$$

$>15$ years: $[1.0-3.5]^{a}$

$[0.8-2.5]$

2.9

Plasma creatine $(\mu \mathrm{mol} / \mathrm{l})$

$$
<10 \text { years: [17-109] }{ }^{a}
$$$$
[3.3-10]
$$$$
59 ; 65
$$

$>10$ years: $[6.0-50]^{a}$

100; 112

Urine GAA/creatinine $(\mathrm{mmol} / \mathrm{mol})$

$$
<15 \text { years: }[2-220]^{a}
$$

$>15$ years: $[3-78]^{a}$
Table 1 Main clinical, biological, genetic and ${ }^{1} H-M R S$ features of the patients affected with PCD (Continued)

Urine creatine/creatinine $(\mathrm{mmol} / \mathrm{mol})$

$\begin{array}{lcc}<4 \text { years: }[6-1208]^{a} & 11 ; 33 & 2762 \\ 4-12 \text { years: }[17-721]^{a} & 18 ; 26 & {[1638-3015]} \\ >12 \text { years: }[11-244]^{a} & {[12-32]} & {[1181-3195]}\end{array}$

Brain ${ }^{1}$ H-MRS Absence of creatine Absence of creatine investigation peak $(n=5) \quad$ peak $(n=6)$

\section{GENETIC DATA}

$\begin{array}{lll}\text { Non-sense mutation } & 2 / 6 & 0 / 11 \\ \text { Missense mutation } & 1 / 6 & 2 / 11 \\ \text { Insertion } & 0 / 6 & 0 / 11 \\ \text { Deletion } & 0 / 6 & 6 / 11 \\ \text { Duplication } & 2 / 6 & 0 / 11 \\ \text { Splicing mutation } & 1 / 6 & 3 / 11\end{array}$

FUNCTIONNAL TESTS

GAMT Activity

(lymphoblasts)

Creatine transport

Deficiency $(2 / 2)$

/

assay (fibroblasts)

Deficit of transport (3/3)

This table groups data from the diagnosed PCD patients and affected siblings. Fraction numbers refer to the number of positive patients (numerator) on the total number of patients studied (denominator) for the indicated item.

Frequencies of signs in patients are expressed as + and - symbols: ++++, presence of signs in all patients; -, absence of signs in all patients; +++, ++ and + , presence of the sign in more than $75 \%$, between 25 to $75 \%$, and less than $25 \%$ of patients, respectively. ${ }^{a}$ Reference laboratory values and age ranges are those published by Verhoeven et al. [38], patient data being expressed as either range or individual values. GAMT, guanidinoacetate methyltransferase; CRTR, creatine transporter SLC6A8.

development and delay at a young age, see [41,42]), and were associated with epilepsy (57\%), motor delay (43\%), hypotonia (43\%) and extrapyramidal symptoms (29\%). Behavioral disturbances were noticed in all patients essentially under the form of attention deficit (100\%) with aggressive behaviors (hetero- and/or auto-mutilating episodes, anger and intolerance to frustration) (86\%), autistic behavior (43\%) and sleeping disturbances (43\%). Most of these signs were usually present before 2 years of age and signs leading to consult are detailed in Table 1.

Diagnosis of GAMT deficiency was established at a median patient age of 5 years, with age values ranging between 2 and 29 years. In urine, the GAA:creatinine ratio (U-GAA/CTN) was increased for all patients and exceeded the upper control limits by 1.8- to 7.2-fold whereas the creatine:creatinine ratio (U-CT/CTN) was normal. When assayed, plasma GAA levels were systematically increased and creatine levels were below or equal to the lowest values of control limits. Cerebral ${ }^{1} \mathrm{H}$-MRS was performed in 5 patients and showed that creatine was undetectable in the brain in all the cases. In 2 of the 7 patients, GAMT activity was measured in lymphoblasts and these enzyme studies validated GAMT 
deficiency. The GAMT gene was studied in 6 of the 7 patients. In each case, patients had presumed (parents not available for study) or confirmed (parents were heterozygous for the mutation) homozygous mutations (Table 2). Mutations were either missense $(n=1)$, nonsense $(n=2)$, frame shift $(n=2)$ or splicing $(n=1)$ mutations.

\section{Patients with SLC6A8 deficiency}

Twelve patients from 10 unrelated families were identified with SLC6A8 deficiency. The sex ratio (M/F) was 11 . When available, the notion of consanguinity was absent. Obstetrical history was available for one patient with a gestational term at 39 weeks; birth weight, 3080 g; length, $48 \mathrm{~cm}$, head circumference, $32 \mathrm{~cm}$. At diagnosis, intellectual disability (100\%) associated with language (75\%) or motor $(83 \%)$ delays were reported in most of the patients. Behavioral abnormalities were observed in $83 \%(10 / 12)$ patients and evoked those seen in GAMT deficiency patients, notably attention deficit (83\%, 10/12 patients) with hyperactivity, autistic behavior (17\%) and sleeping disturbances (8\%). No aggressive behavior was observed. Epilepsy was reported in $17 \%$ of the patients. The main signs leading parents to consult (see Table 1 for an overview) were combined motor and speech delays; autistic behavior was the second cause of consultation. Familial inquiries led to the diagnosis of SLC6A8 deficiency in 2 of the 12 patients having an affected sibling. Anamnesis indicated that the initial signs could be present before the age of 2 years with in particular global psychomotor retardation and to a lesser extent epilepsy and autistic behavior. The median age for diagnosis was 9.5 years with age values ranging between 2.5 and 28 years.

The U-CT/CTN ratio was increased in all male patients, and exceeded the upper control limit by 2.3- to 13.6-fold. For the affected girl, the U-CT/CTN ratio was only 2.3-fold greater than the upper control limit. In plasma, creatine levels were normal or moderately increased (up to 1.3-fold greater than the upper control limit value). Plasma GAA levels were normal (or slightly affected), as were urinary U-GAA/CTN ratios. Cerebral
${ }^{1} \mathrm{H}$-MRS was performed in $50 \%(6 / 12)$ of the patients and consistently confirmed creatine deficiency in the brain in all examined patients (undetectable creatine peak). In 3 of the 12 (25\%) patients, a functional study of SCL6A8 activity was carried out in cultured skin fibroblasts, and evidenced a deficiency of the creatine transporter function.

The SCL6A8 gene was studied in 92\% (11/12) of the patients (Table 3 ). A hemizygous mutation was identified for all the affected male patients. For the female patient, a pathogenic mutation was identified at a heterozygous state and no $\mathrm{X}$-inactivation biases could be identified in leucocytes. Mutations were either missense $(n=2)$, frame shift $(n=6)$ or splicing $(n=3)$ mutations. The underlying mutations were also detected in the mothers of the patients, except for the heterozygous female patient (c.942_944delCTT in exon 6) and a hemizygous boy (c.1208C > A in exon 8). In the latter two patients, SLC6A8 deficiencies were concluded to result from neomutation, germinal or somatic mosaicism.

\section{Discussion}

In the present study we have identified 16 new patients diagnosed with a PCD over a period of 28 months, as well as three affected siblings. Intellectual disability and attention deficit were, along with motor and speech delays, the main symptoms reported by the medical staff involved in the diagnosis of these disorders. Nevertheless, it is surprising that intellectual disability, which was a sign observed by the medical examiners in all patients with PCD, was not the main cause leading the parents to consult. This might reflect the difficulty of identifying intellectual disability in the very young child or in turn to of associating intellectual disability with PCD. These disorders are known to progress via irreversible lesions of the brain, so therapeutic measures are needed to be taken early in the life of the patients. For GAMT patients, supplementation with creatine together with a GAA lowering strategy (ornithine supplementation with or without arginine restriction) may represent an efficient treatment strategy [43-46] and may provide some medical neurological benefit for GAMT patients. In

Table 2 Mutations in the GAMT gene

\begin{tabular}{|c|c|c|c|c|c|}
\hline \multicolumn{3}{|c|}{ Mutations } & \multicolumn{2}{|c|}{ Number of patients identified in this study } & \multirow[t]{2}{*}{ Reference } \\
\hline Exon (e)/Intron (i) & Nucleotide & Amino acid & Homozygous & Heterozygous & \\
\hline e2 & c. $289 \mathrm{C}>\mathrm{T}$ & p.Q97X & 1 & - & This study \\
\hline e2 & c.299_311dup13 & p.R105GfsX26 & $2^{a}$ & - & Dhar et al., 2009 [27] \\
\hline i3 & $c .391+15 G>T$ & p.(?) & 1 & - & This study \\
\hline e5 & C. $506 \mathrm{G}>\mathrm{A}$ & p.C169Y & 1 & - & Caldeira Araujo et al., 2005 [63] \\
\hline e6 & c. $577 \mathrm{C}>\mathrm{T}$ & p.Q193X & 1 & - & This study \\
\hline
\end{tabular}

Nucleotide numbering starting at the first adenine of the translation initiation codon ATG.

${ }^{a}$ Two affected siblings. 
Table 3 Mutations in the SLC6A8 gene

\begin{tabular}{|c|c|c|c|c|c|}
\hline \multicolumn{3}{|c|}{ Mutations } & \multicolumn{2}{|c|}{ Number of patients identified in this study } & \multirow[t]{3}{*}{ Reference } \\
\hline Exon (e)/Intron (i) & Nucleotide & Amino acid & Male & Female & \\
\hline & & & Hemizigous & Heterozygous & \\
\hline e2 & c.321_323delCTT & p.F107del & $2^{a}$ & - & Degrauw et al., 2002[64] \\
\hline$i 4$ & c. $778-2 A>G$ & p.(?) & 1 & - & Betsalel et al., 2011[61] \\
\hline e6 & C. $926 C>A$ & p.A309E & 1 & - & This study \\
\hline e6 & c.930delG & p.T311PfsX85 & 1 & - & This study \\
\hline e6 & c.942_944delCTT & p.F314del & - & 1 & Fons et al., 2009[65] \\
\hline e6 & c.1006_1008delAAC & p.N336del & 1 & - & Clark et al., 2006[56] \\
\hline e8 & c. $1208 C>A$ & p.A403D & 1 & - & This study \\
\hline i9 & c.1393-1G > A & p.(?) & $2^{a}$ & - & This study \\
\hline e11 & c.1519_1543del & p.1507LfsX5 & 1 & - & Betsalel et al., 2011[61] \\
\hline
\end{tabular}

Nucleotide numbering starting at the first adenine of the translation initiation codon ATG.

a Two affected brothers.

these conditions, patients may recover a normal brain creatine peak as detected by ${ }^{1} \mathrm{H}$-MRS as well as normal urine GAA levels. They also improve clinically with better language and social development and apparently some regression of the epilepsy [47]. By contrast, in patients with SLC6A8 deficiency, supplementations with creatine and its precursors arginine or glycine, though improving muscular signs, were found to be without substantial benefit on cognitive and psychiatric signs, and failed to modify the creatine signal in ${ }^{1} \mathrm{H}$-MRS imaging of the brain [48], suggesting the inefficacy of these supplementations in improving brain as stated by other studies $[4,19,30,49-52]$. Though there is hope to treat efficiently this group of patients through the discovery of creatine pro-drugs capable of entering the brain and cells via a by-pass of the SCL6A8 creatine transporter, some clinical studies also question the inefficacy of the therapeutic measures mentioned above and, on the contrary, show beneficial effects with creatine and/or arginine supplementations in patients with SLC6A8 deficiency. In a heterozygous female patient with intractable epilepsy, treatment with creatine combined with arginine and glycine completely resolved seizures [53]. Creatine supplementation was also described to improve the neurological, language and behavioral status and was associated with a rise in the brain creatine peak as demonstrated by MRS in a child with SLC6A8 deficiency [54]. In a recent study, creatine deficient patients were also shown to be improved by a L-arginine-based therapy which positively impacted daily living skills, lowered the frequency of epileptic episodes and induced a mild increase in brain creatine and phosphocreatine MRS signals although normal cerebral levels of these metabolites were not recovered [55].

The calculated prevalence of PCD in our cohort was $0.25 \%$. This result was not expected because most studies report a higher prevalence of PCD, notably SLC6A8 deficiency, which is generally estimated between $1 \%$ and $3 \%$ of the population affected with intellectual disability [56-59]. However, this prevalence has been considered to be an overestimation of the real prevalence of PCD. Because PCDs are monogenic disorders, their prevalence was proposed to be no different from that of other nonsyndromic diseases such ARX (Aristaless-Related homeobox gene located on $\mathrm{X}$ chromosome) and therefore closer to $0.1 \%-0.3 \%$ [60]. Thus, for the first time, our screening study provides strong practice-based evidence confirming this estimated prevalence for PCD. The girl diagnosed with SLC6A8 deficiency presented a severe phenotype similar to affected male patients, an observation that is not surprising in view of recent work $[34,61,62]$ also describing this X-linked disorder in the female population with intellectual disability. In this respect, screening for SLC6A8 deficiency in female patients should be included in the diagnostic workup since this disorder still remains under-diagnosed. However, it should be noted that if the urinary $\mathrm{U}-\mathrm{CT} / \mathrm{CTN}$ ratio is not increased the diagnosis cannot be ruled out because it has been shown that the majority of female patients with a heterozygous mutation in SLC6A8 have a normal ratio, although the average ratio of this group is increased [62]. It might be recommended to perform brain MRS and/or SLC6A8 gene studies in female patients with suspected creatine transporter deficiency.

Five mutations were identified for GAMT gene in 6 unrelated families. These included 3 new mutations, c. $289 \mathrm{C}>\mathrm{T}$, c. $391+15 \mathrm{G}>\mathrm{T}$ and c. $577 \mathrm{C}>\mathrm{T}$, and two previously described mutations, c.299_311dup13 [27] and c.506G > A [63] (Table 2). The new mutation c391+ $15 \mathrm{G}>\mathrm{T}$ was considered pathogenic by the creation of an alternative splicing donor site in intron 3 (Alamut 
software), by the segregation of this mutation in his parents and because it was linked with decreased GAMT activity in cultured fibroblasts.

The SLC6A8 gene was analyzed in 11 patients from 9 unrelated families. Nine mutations were identified throughout the gene, including 4 new mutations ( 2 missense (c.1208C >A and c.926C >A), 1 frameshift (c.930delG) and 1 splicing (c.1393-1G > A) mutation), and 5 previously described mutations (c.321_323delCTT [64], c.942_944delCTT [65], c.1006_1008delAAC [56], c.778-2A > G and c.1519_1543del [61]) (Table 3). In accordance with the results of Clark et al. [56], we found a fairly high proportion of frameshift and splicing mutations in our patients, and approximately one fifth of the mutations of SLC6A8, but not GAMT, were attributed to neo-mutation, germinal or somatic mosaicism events.

Interestingly, DNA studies performed in our patients clearly showed that mutations were essentially patient/ family specific and highlighted the absence of hot spot mutations in these genes. Finally, as a mutation was identified in each patient for whom DNA was analyzed, the study of the target gene appears to be a key diagnostic step for PCD in suspect patients, keeping in mind that secondary creatine disorders such as deficiencies of ornithine delta-aminotransferase (EC 2.6.1.13) [66,67], urea [68-71] and homocysteine/ methionine $[72,73]$ cycles, and succinate semialdehyde dehydrogenase (SSADH) (EC 1.2.1.24) [74,75] may also be alternative causes of abnormal laboratory values of creatine metabolism markers.

\section{Conclusion}

Screening for PCD in the population with neurological disorders of unknown etiology has shown that these patients express important clinical signs of PCD which mainly include intellectual disability, speech delay, epilepsy, attention deficit and autistic behavior.

In this respect, some improvement might be made by conducting an information campaign among general and specialized educational staff to contribute to better recognizing intellectual disability and hence earlier referral of the child for professional medical evaluation. Improved anamnesis in regard to the familial history of possible patients might be also helpful. As suggested in the present study and highlighted in other work, both female and male patients can be concerned by severe PCD. However, and importantly, our study has shown that the prevalence of PCD remains low in the patients with unexplained neurological symptoms. Finally, the mutational spectrum of PCD has been extended by the new mutations detected during the present screening effort.

\section{Consent}

When PCD was suspected informed consent was obtained from patients and/or their parents for the DNA analysis of GAMT or SLC6A8 genes.

\section{Additional file}

\begin{abstract}
Additional file 1: Updated view of creatine metabolism and transport. Two enzymes are involved in the biosynthesis of creatine: arginine-glycineamidinotransferase (AGAT) and guanidinoacetatemethyltransferase (GAMT). A third protein, a plasma membrane transporter (SLC6A8), supplies the cell with creatine. A deficiency of any of these three biosynthesis and transport steps (namely, AGAT deficiency, GAMT deficiency and creatine transporter deficiency) leads to a deficiency of creatine in the brain (collapse of brain content in creatine). Biosynthesis of creatine happens in organs that contain both AGAT and GAMT. However, particularly in the brain, AGAT and GAMT may be distributed in different cells, so the guanidinoacetate (GAA) formed in one cell can be further transformed into creatine in another cell. This requires, as illustrated in the figure, the creatine transporter which can supply cells not only with creatine but also with GAA. The reason for collapse in brain creatine in either AGAT or GAMT deficiency is easily understood by a severe impairment of one of the two creatine biosynthesis steps. In creatine transporter deficiency, several facts concur including : (i) heterogeneity of brain cells for their content in AGAT and GAMT, (ii) most cerebral cells contain only of one of these two creatine biosynthesis enzymes; (iii) the blood-brain barrier is weakly permeable to systemic creatine and the brain must secure most of its creatine requirements by endogenous synthesis; and (iv) the creatine transporter contributes in the brain not only to supplying the cells with creatine but also directly to creatine biosynthesis by bridging the formation of an intermediate (GAA) in one cerebral cell to its utilization (towards creatine synthesis) in another cerebral cell. Another protein, the taurine transporter has also been shown to be active on GAA but in this case it leads to its removal from brain structures and CSF into blood, a feature having GAA detoxifying properties, notably in GAMT deficiency. Also depicted in the figure is the creatine kinase (CK) system (based on phosphocreatine as a stored form of energy produced from and capable of restoring ATP) and the non-enzymatic formation from creatine and phosphocreatine of creatinine (an end product of creatine metabolism removed in body fluids). Not depicted in the figure is the transit via body fluids which may take place in intercellular transfer and removal of creatine metabolites. For additional considerations and references, the reader is kindly referred to the recent review of Olivier Braissant (Ref [14] in the text).
\end{abstract}

\section{Competing interests}

The authors declare that they have no competing interests.

\section{Authors' contributions}

DC and MJCC contributed equally to this work and are first co-authors. Authors (DC, MJCC, GB, KMM, DD, JMC; AC, W; JFB; JMP, GSI, OD, AA, LL, VD, $M G, R D, N P, J V$ ) working in the six major French university hospitals (including those of Lille, Lyon and Angers and the Hôpital Necker Enfants Malades, Hôpital Robert Debré and Hôpital Raymond Poincaré from the Public Assistance of Parisian Hospitals) collaborated to group and to generate clinical biological, biochemical and genetic data. The other authors were also involved in the clinical follow-up (AG, GP, BC, FR, KD, MT) or biological chemistry (SB) of some of the patients and in the gene studies (FC, FP, DH and GSA). DC, MJCC, GB, NP and JV analyzed and integrated the clinical and paraclinical data. JV coordinated the writing of the manuscript with DC, MJCC and GB. All authors read and approved the final manuscript.

\section{Acknowledgments}

We would like to thank the patients for participating in this study. This work was supported by grants from the French Ministère de la Santé (PHRC 2003R/1903) and FMO (Fédération des Maladies Orphelines). 


\section{Author details}

${ }^{1}$ Hospices Civils de Lyon, Service Maladies Héréditaires du Métabolisme et Dépistage Néonatal, Groupement Hospitalier Est, Bron 69677, France. 2Département de Biochimie et Biologie Moléculaire, Laboratoire d'Hormonologie, Métabolisme-Nutrition \& Oncologie (HMNO)-Centre de Biologie et Pathologie (CBP) Pierre-Marie Degand, CHRU Lille, Lille 59037, France. ${ }^{3}$ Mass Spectrometry Application Laboratory, University of Lille 2, Lille 59045, France. ${ }^{4}$ Metabolic Unit, Department of Clinical Chemistry, VU University Medical Center Amsterdam, Amsterdam, The Netherlands. ${ }^{5}$ Centre de Référence des Maladies Héréditaires du Métabolisme, Hôpital Jeanne de Flandres, CHRU Lille, Lille 59037, France. 'Service de Neurologie Infantile, Hôpital Roger Salengro, CHRU Lille, Lille 59037, France. ${ }^{7}$ Service de neurologie pédiatrique, CHU de Lyon-GH Est - Hôpital Femme Mère Enfant, Bron Cedex 69677, France. ${ }^{8}$ Laboratory of Biochemistry, Necker - Enfants Malades Hospital and Université Paris Descartes, Paris 75015, France. ${ }^{9}$ Centre de Référence des Maladies Héréditaires du Métabolisme, Hôpital Necker des Enfants Malades and Université Paris Descartes, 149 rue de Sèvres, Paris 75015, France. ${ }^{10}$ Département de Biochimie-Hormonologie, CHU Hôpital Robert Debré, Paris 75019, France. ${ }^{11}$ Unité de Neurologie Pédiatrique, Département de Pédiatrie, Hôpital Raymond Poincare, Paris-IdF-Ouest University, Paris, France. ${ }^{12}$ Laboratoire de Biochimie et Biologie Moléculaire, CHU Angers, Angers 49033, France. ${ }^{13}$ Service de Neuropédiatrie - CHU de Bicêtre, Le Kremlin Bicêtre Cedex 94275, France. ${ }^{14}$ Service de Neuropédiatrie, Hôpital Armand Trousseau, Groupement hospitalier universitaire Est, Paris 75012, France. ${ }^{15}$ Unité Fonctionnelle de Génétique Médicale AP-HP, Département de Génétique et Cytogénétique, Centre de Référence « Déficiences intellectuelles de causes rares », CRicm, UMR-S975, Groupe Hospitalier Pitié-Salpêtrière, Paris F-75013, France. ${ }^{16}$ Neuropédiatrie, CHRU Montpellier, \& Inserm U1046, Université Montpellier 1 \& 2, Montpellier Cedex 5 34295, France. ${ }^{17}$ Service Neuropédiatrie, AP-HM Hôpital de la Timone, Marseille Cedex 5 13385, France. ${ }^{18}$ Service de Génétique, CHU de SaintÉtienne Hôpital Nord, Saint-Etienne Cédex 2 42055, France. ${ }^{19}$ Service de génétique Centre hospitalier Felix Guyon (Saint-Denis) Bellepierre, Saint-Denis cedex 97405 , France. ${ }^{20}$ Service de Neuropédiatrie, Hôpital de l'Archet 2, Nice Cedex 3 06202, France. ${ }^{21}$ Service de Génétique Médicale, CHU Ch. Nicolle, Rouen Cedex 76031, France. ${ }^{22}$ Institut de Biologie Clinique, CHU Ch. Nicolle, Rouen Cedex 76031, France. ${ }^{23}$ Service de Génétique, CHU Clémenceau, Caen 14033, France. ${ }^{24}$ Service de Pédopsychiatrie CHU Hôpital Robert Debré, Paris 75019, France. ${ }^{25}$ Service de Neuropédiatrie - CHU de Bicêtre, Le Kremlin Bicêtre Cedex 94275, France. ${ }^{26}$ Inserm, Laboratoire Externe, Département du Prof. Nicole Porchet, HMNO, Centre de Biologie et Pathologie (CBP) PierreMarie Degand, CHRU Lille, Lille 59037, France.

Received: 21 May 2012 Accepted: 7 December 2012 Published: 13 December 2012

\section{References}

1. Chevreul E: Sur la composition chimique du bouillon de viandes (On the chemical composition of meatbroth). J Pharm Sci Access 1835, 21:231-242.

2. Balsom $P$, Söderland $K$, Ekbolm B: Creatine in humans with special reference to creatine supplementation. Sports Med 1994, 18(4):268-280.

3. Wyss M, Kaddurah-Daouk R: Creatine and creatinine metabolism. Physiol Rev 2000, 80(3):1107-1213.

4. Wyss M, Schulze A: Health implications of creatine: can oral creatine supplementation protect against neurological and atherosclerotic disease? Neuroscience 2002, 112(2):243-260.

5. Lundsgaard $\mathrm{E}$ : Untersuchungen über Muskel kontraktion en ohne Milchsäure bildung (Muscle contraction without formation of lactic acid). Biochem Z 1930, 217:162-177.

6. Lundsgaard E: Weitere Untersuchungen fiber Muskel kontraktion en ohne Milchsäure bildung. (Further investigation on muscle contraction without lactate formation). Biochem Z 1930, 227:51-83.

7. Lohman K: Über die enzymatische aufspaltung der kreatin phosphorsaure; zugleich ein beitrag zum chemismus der muskel kontraktion. (On the enzymic cleavage of creatinephosphate; also a contribution to the chemistry of the muscle contraction). Biochem $Z$ 1934, 271:264-277.

8. Engelhardt WA, Lyubimova MN: Myosin and adenosine triphosphatase Nature 1939, 144:668-669.
9. Andres RH, Ducray AD, Schlattner U, Wallimannb T, Widmer HF: Functions and effects of creatine in the central nervous system. Brain Res Bull 2008 76(4):329-343

10. Braissant $\mathrm{O}$, Henry $\mathrm{H}$ : AGAT, GAMT and SLC6A8 distribution in the central nervous system, in relation to creatine deficiency syndromes: A review. J Inherit Metab Dis 2008, 31(2):230-239.

11. Braissant $O$, Béard $E$, Torrent $C$, Henry H: Dissociation of AGAT, GAMT and SLC6A8 in CNS: relevance to creatine deficiency syndromes. Neurobiol Dis 2010, 37(2):423-433.

12. Béard E, Braissant O: Synthesis and transport of creatine in the CNS: importance for cerebral functions. J Neurochem 2010, 115(2):297-313.

13. Braissant $\mathrm{O}$, Henry $H$, Loup $M$, Eilers B, Bachmann C: Endogenous synthesis and transport of creatine in the rat brain: an in situ hybridization study. Mol Brain Res 2001, 86(1-2):193-201.

14. Braissant O: Creatine and guanidinoacetate transport at blood-brain and blood-cerebrospinal fluid barriers. J Inherit Metab Dis 2012, 35(4):655-664

15. Schulze A, Battini R: Pre-symptomatic treatment of creatine biosynthesis defects. Subcell Biochem 2007, 46:167-181.

16. Nasrallah F, Feki M, Kaabachi N: Creatine and creatine deficiency syndromes: biochemical and clinical aspects. Pediatr Neurol 2010, 42(3):163-171

17. Schulze A: Creatine deficiency syndromes. Mol Cell Biochem 2003, 244(1-2):143-150.

18. Stockler S, Schutz PW, Salomons GS: Cerebral creatine deficiency syndromes: clinical aspects, treatment and pathophysiology. Subcell Biochem 2007, 46:149-166.

19. Stromberger C, Bodamer OA, Stöckler-Ipsiroglu S: Clinical characteristics and diagnostic clues in inborn errors of creatine metabolism. J Inherit Metab Dis 2003, 26(2-3):299-308.

20. Sykut-Cegielska J, Gradowska W, Mercimek-Mahmutoglu S, Stöckler-Ipsiroglu S: Biochemical and clinical characteristics of creatine deficiency syndromes. Acta Biochim Pol 2004, 51(4):875-882.

21. Battini R, Alessandrì MG, Leuzzi V, Moro F, Tosetti M, Bianchi MC, Cioni G: Arginine: glycine amidinotransferase (AGAT) deficiency in a newborn: early treatment can prevent phenotypic expression of the disease. J Pediatr 2006, 148(6):828-830.

22. Carducci C, Birarelli M, Leuzzi V, Carducci C, Battini R, Cioni G, Antonozzi I: Guanidinoacetate and creatine plus creatinine assessment in physiologic fluids: an effective diagnostic tool for the biochemical diagnosis of arginine:glycine amidinotransferase and guanidinoacetate methyltransferase deficiencies. Clin Chem 2002, 48(10):1772-1778.

23. Item CB, Stöckler-Ipsiroglu S, Stromberger C, Mühl A, Alessandrì MG, Bianchi MC, Tosetti M, Fornai F, Cioni G: Arginine:glycine amidinotransferase deficiency: the third inborn error of creatine metabolism in humans. Am J Hum Genet 2001, 69(5):1127-1133.

24. Mercimek-Mahmutoglu S, Stöckler-lpsiroglu S: Creatine deficiency syndromes. In GeneReviews. Edited by Pagon RA, Bird TC, Dolan CR, Stephens K. Seattle (WA): University of Washington, Seattle; 2009. 1993-2010.

25. Sun Q, O'Brien WE: Diagnosis of creatine metabolism disorders by determining creatine and guanidinoacetate in plasma and urine. Methods Mol Biol 2010, 603:175-185.

26. Bodamer OA, Bloesch SM, Gregg AR, Stockler-lpsiroglu S, O'Brien WE: Analysis of guanidinoacetate and creatine by isotope dilution electrospray tandem mass spectrometry. Clin Chim Acta 2001, 308(1-2):173-178

27. Dhar SU, Scaglia F, Li FY, Smith L, Barshop BA, Eng CM, Haas RH, Hunter JV, Lotze T, Maranda B, Willis M, Abdenur JE, Chen E, O'Brien W, Wong L: Expanded clinical and molecular spectrum of guanidinoacetatemethyltransferase (GAMT) deficiency. Mol Genet Metab 2009, 96(1):38-43.

28. Alcaide P, Merinero B, Ruiz-Sala P, Richard E, Navarrete R, Arias A, Ribes A, Artuch R, Campistol J, Ugarte M, Rodríguez-Pombo P: Defining the pathogenicity of creatine deficiency syndrome. Hum Mutat 2011, 32(3):282-291.

29. Almeida LS, Verhoeven NM, Roos B, Valongo C, Cardoso ML, Vilarinho L, Salomons GS, Jakobs C: Creatine and guanidinoacetate: diagnostic markers for inborn errors in creatine biosynthesis and transport. Mol Genet Metab 2004, 82(3):214-219. 
30. Bizzi A, Bugiani M, Salomons GS, Hunneman DH, Moroni I, Estienne M, Danesi U, Jakobs C, Uziel G: X-linked creatine deficiency syndrome: a novel mutation in creatine transporter gene SLC6A8. Ann Neurol 2002, 52(2):227-231

31. Mercimek-Mahmutoglu S, Muehl A, Salomons GS, Neophytou B, Moeslinger D, Struys E, Bodamer OA, Jakobs C, Stockler-lpsiroglu S: Screening for X-linked creatine transporter (SLC6A8) deficiency via simultaneous determination of urinary creatine to creatinine ratio by tandem mass-spectrometry. Mol Genet Metab 2009, 96(4):273-275.

32. Salomons GS, van Dooren SJ, Verhoeven NM, Cecil KM, Ball WS, Degrauw TJ, Jakobs C: X-linked creatine-transporter gene (SLC6A8) defect: a new creatine-deficiency syndrome. Am J Hum Genet 2001, 68(6):1497-1500

33. Sempere $A$, Fons $C$, Arias A, Rodríguez-Pombo P, Colomer R, Merinero B, Alcaide P, Capdevila A, Ribes A, Artuch R, Campistol J: Creatine transporter deficiency in two adult patients with static encephalopathy. J Inherit Metab Dis 2009. doi:Short Report \#, 158 10.1007/s10545-009-1083-2.

34. Arias A, Corbella M, Fons C, Sempere A, García-Villoria J, Ormazabal A, Poo P, Pineda M, Vilaseca MA, Campistol J, Briones P, Pàmpols T, Salomons GS, Ribes A, Artuch R: Creatine transporter deficiency: prevalence among patients with mental retardation and pitfalls in metabolite screening. Clin Biochem 2007, 40(16-17):1328-1331.

35. Cognat S, Cheillan D, Piraud M, Roos B, Jakobs C, Vianey-Saban C: Determination of guanidinoacetate and creatine in urine and plasma by liquid chromatography-tandem mass spectrometry. Clin Chem 2004 50(8):1459-1461.

36. Jaffe M: Uber den niederschlag, welchen pikrinsaure in normalen ham erzeugt und ubereineneue reaction des kreatinins. Z Physiol Chem 1886, 10:391-400

37. Tsikas D, Wolf A, Mitschke A, Gutzki FM, Will W, Bader M: GC-MS determination of creatinine in human biological fluids as pentafluorobenzyl derivative in clinical studies and biomonitoring: Inter-laboratory comparison in urine with Jaffé, HPLC and enzymatic assays. J Chromatogr B Analyt Technol Biomed Life Sci 2010, 878(27):2582-2592

38. Verhoeven NM, Salomons GS, Jakobs C: Laboratory diagnosis of defects of creatine biosynthesis and transport. Clin Chim Acta 2005, $361(1-2): 1-9$.

39. Verhoeven NM, Roos B, Struys EA, Salomons GS, Van Der Knaap MS, Jakobs C: Enzyme assay for diagnosis of guanidinoacetatemethyltransferase deficiency. Clin Chem 2004, 50(2):441-443.

40. Fokkema IF, Taschner PE, Schaafsma GC, Celli J, Laros JF, den Dunnen JT: LOVD v.2.0: the next generation in gene variant databases. Hum Mutat 2011, 32(5):557-563.

41. Lung AK, Kao CP: Evaluation and management of the child with speech delay. Am Fam Physician 1999, 59(11):3121-3128.

42. Wankoff LS: Warning signs in the development of speech, language, and communication: when to refer to a speech-language pathologist. $J$ Child Adolesc Psychiatr Nurs 2011, 24(3):175-184

43. Bianchi MC, Tosetti M, Battini R, Leuzzi V, Alessandri MG, Carducci C, Antonozzi l, Cioni G: Treatment monitoring of brain creatine deficiency syndromes: a 1H- and 31P-MR spectroscopy study. Am J Neuroradiol 2007 , 28(3):548-554

44. Ensenauer R, Thiel T, Schwab KO, Tacke U, Stöckler S: Guanidinoacetate methyltransferase deficiency: differences of creatine uptake in human brain and muscle. Mol Genet Metab 2004, 82(3):208-213.

45. Gordon N: Guanidinoacetate methyltransferase deficiency (GAMT). Brain Dev 2010, 32(2):79-81.

46. Schulze A, Hoffmann GF, Bachert P, Kirsch S, Salomons GS, Verhoeven NM, Mayatepek E: Presymptomatic treatment of neonatal guanidinoacetate methyltransferase deficiency. Neurology 2006, 67(4):719-721.

47. Blanc E: Approche thérapeutique d'une maladie génétique rare le déficit de synthèse en créatine par déficit en guanidinoacétate méthyltransferase. Thèse d'Exercice de Pharmacie (Cheillan D, Thesis Director) 2009, Université Claude Bernard (Lyon, France), http://www.sudoc.fr/ 145878910, 82 pages.

48. Valayannopoulos V, Boddaert N, Chabli A, Barbier V, Desguerre I, Philippe A, Afenjar A, Mazzuca M, Cheillan D, Munnich A, de Keyzer Y, Jakobs C, Salomons GS, de Lonlay P: Treatment by oral creatine, L-arginine and L-glycine in six severely affected patients with creatine transporter defect. J Inherit Metab Dis 2012, 35(1):151-157.
49. Braissant $\mathrm{O}$, Henry $\mathrm{H}$, Béard $\mathrm{E}$, Uldry J: Creatine deficiency syndromes and the importance of creatine synthesis in the brain. Amino Acids 2011, 40(5):1315-1324

50. Evangeliou A, Vasilaki K, Karagianni P, Nikolaidis N: Clinical applications of creatine supplementation on paediatrics. Curr Pharm Biotechnol 2009, 10(7):683-690

51. Fons C, Sempere A, Arias A, López-Sala A, Póo P, Pineda M, Mas A, Vilaseca MA, Salomons GS, Ribes A, Artuch R, Campistol J: Arginine supplementation in four patients with X-linked creatine transporter defect. J Inherit Metab Dis 2008, 31(6):724-728.

52. Salomons GS, van Dooren SJ, Verhoeven NM, Marsden D, Schwartz C, Cecil KM, De Grauw TJ, Jakobs C: X-linked creatine transporter defect: an overview. J Inherit Metab Dis 2003, 26(2-3):309-318.

53. Mercimek-Mahmutoglu S, Connolly MB, Poskitt KJ, Horvath GA, Lowry N, Salomons GS, Casey B, Sinclair G, Davis C, Jakobs C, Stockler-lpsiroglu S: Treatment of intractable epilepsy in a female with SLC6A8 deficiency. Mol Genet Metab 2010, 101(4):409-412.

54. Chilosi A, Leuzzi V, Battini R, Tosetti M, Ferretti G, Comparini A, Casarano M, Moretti E, Alessandri MG, Bianchi MC, Cioni G: Treatment with L-arginine improves neuropsychological disorders in a child with creatine transporter defect. Neurocase 2008, 14(2):151-161.

55. Chilosi A, Casarano M, Comparini A, Battaglia FM, Mancardi MM, Schiaffino C, Tosetti M, Leuzzi V, Battini R, Cioni G: Neuropsychological profile and clinical effects of arginine treatment in children with creatine transport deficiency. Orphanet J Rare Dis 2012, 7(1):43.

56. Clark AJ, Rosenberg EH, Almeida LS, Wood TC, Jakobs C, Stevenson RE, Schwartz CE, Salomons GS: X-linked CT (SLC6A8) mutations in about 1\% of males with mental retardation of unknown etiology. Hum Genet 2006 119(6):604-610.

57. Lion-François L, Cheillan D, Pitelet G, Acquaviva-Bourdain C, Bussy G, Cotton F, Guibaud L, Gérard D, Rivier C, Vianey-Saban C, Jakobs C, Salomons GS, des Portes $V$ : High frequency of creatine deficiency syndromes in patients with unexplained mental retardation. Neurology 2006, 67(9):1713-1714.

58. Newmeyer A, Cecil KM, Schapiro M, Clark JF, Degrauw TJ: Incidence of brain $\mathrm{CT}$ deficiency in males with developmental delay referred for brain magnetic resonance imaging. J Dev Behav Pediatr 2005, 26(4):276-282.

59. Rosenberg EH, Almeida LS, Kleefstra T, De Grauw RS, Yntema HG, Bahi N, Moraine C, Ropers HH, Fryns JP, De Grauw TJ, Jakobs C, Salomons GS: High prevalence of SLC6A8 deficiency in X-linked mental retardation. Am J Hum Genet 2004, 75(1):97-105.

60. Mandel JL: Comparative frequency of fragile-X (FMR1) and creatine transporter (SLC6A8) mutations in X-linked mental retardation. Am J Hum Genet 2004, 75(4):730-731.

61. Betsalel OT, Rosenberg EH, Almeida LS, Kleefstra T, Schwartz CE, Valayannopoulos $V$, Abdul-Rahman O, Poplawski N, Vilarinho L, Wolf P, den Dunnen JT, Jakobs C, Salomons GS: Characterization of novel SLC6A8 variants with the use of splice-site analysis tools and implementation of a newly developed LOVD database. Eur J Hum Genet 2011, 19(1):56-63.

62. van de Kamp JM, Mancini GM, Pouwels PJ, Betsalel OT, van Dooren SJ, de Koning I, Steenweg ME, Jakobs C, van der Knaap MS, Salomons GS: Clinical features and $X$-inactivation in females heterozygous for creatine transporter defect. Clin Genet 2011, 79(3):264-272.

63. Caldeira Araújo H, Smit W, Verhoeven NM, Salomons GS, Silva S, Vasconcelos R, Tomás H, Tavares De Almeida I, Jakobs C, Duran M: Guanidinoacetate methyltransferase deficiency identified in adults and a child with mental retardation. Am J Med Genet A 2005, 133A(2):122-127.

64. De Grauw TJ, Salomons GS, Cecil KM, Chuck G, Newmeyer A, Schapiro MB, Jakobs C: Congenital creatine transporter deficiency. Neuropediatrics 2002, 33(5):232-238

65. Fons C, Sempere A, Sanmartí FX, Arias A, Póo P, Pineda M, Ribes A, Merinero B, Vilaseca MA, Salomons GS, Artuch R, Campistol J: Epilepsy spectrum in cerebral creatine transporter deficiency. Epilepsia 2009, 50(9):2168-2170

66. Sipilä I: Inhibition of arginine-glycine amidinotransferase by ornithine. A possible mechanism for the muscular and chorioretinal atrophies in gyrate atrophy of the choroid and retina with hyperornithinemia. Biochim Biophys Acta 1980, 613(1):79-84.

67. Valayannopoulos V, Boddaert N, Mention K, Touati G, Barbier V, Chabli A, Sedel F, Kaplan J, Dufier JL, Seidenwurm D, Rabier D, Saudubray JM, de 
Lonlay P: Secondary creatine deficiency in ornithine deltaaminotransferase deficiency. Mol Genet Metab 2009, 97(2):109-113.

68. Arias A, Garcia-Villoria J, Ribes A: Guanidinoacetate and creatine/creatinine levels in controls and patients with urea cycle defects. Mol Genet Metab 2004, 82(3):220-223.

69. Braissant O: Ammonia toxicity to the brain: effects on creatine metabolism and transport and protective roles of creatine. Mol Genet Metab 2010, 100(Suppl 1):S53-S58.

70. Brosnan JT, Brosnan ME: Creatine metabolism and the urea cycle. Mol Genet Metab 2010, 100(Suppl 1):S49-S52.

71. Roze E, Azuar C, Menuel C, Haberle J, Guillevin R: Usefulness of magnetic resonance spectroscopy in urea cycle disorders. Pediatr Neurol 2007, 37(3):222-225.

72. Baric I, Fumic K, Glenn B, Cuk M, Schulze A, Finkelstein JD, James SJ, Mejaski-Bosnjak V, Pazanin L, Pogribny IP, Rados M, Sarnavka V, Scukanec-Spoljar M, Allen RH, Stabler S, Uzelac L, Vugrek O, Wagner C, Zeisel S, Mudd SH: S-adenosylhomocysteine hydrolase deficiency in a human: a genetic disorder of methionine metabolism. Proc Natl Acad Sci USA 2004, 101(12):4234-4239.

73. Bodamer OA, Sahoo T, Beaudet AL, O'Brien WE, Bottiglieri T, StocklerIpsiroglu S, Wagner C, Scaglia F: Creatine metabolism in combined methylmalonicaciduria and homocystinuria. Ann Neurol 2005, 57(4):557-560.

74. Jansen EE, Verhoeven NM, Jakobs C, Schulze A, Senephansiri H, Gupta M, Snead OC, Gibson KM: Increased guanidino species in murine and human succinate semialdehyde dehydrogenase (SSADH) deficiency. Biochim Biophys Acta 2006, 1762(4):494-498.

75. Vamecq J, Joncquel-Chevalier Curt M, Mention-Mulliez K, Dobbelaere D, Briand G: Rise in brain GABA to further stress the metabolic link between valproate and creatine. Mol Genet Metab 2011, 102(2):232-234.

doi:10.1186/1750-1172-7-96

Cite this article as: Cheillan et al:: Screening for primary creatine deficiencies in French patients with unexplained neurological symptoms. Orphanet Journal of Rare Diseases 2012 7:96.

\section{Submit your next manuscript to BioMed Central and take full advantage of:}

- Convenient online submission

- Thorough peer review

- No space constraints or color figure charges

- Immediate publication on acceptance

- Inclusion in PubMed, CAS, Scopus and Google Scholar

- Research which is freely available for redistribution 